Z. klin. Chem. u. klin. Biochem.

8. Jg., S. 38-40, Januar 1970

\title{
Isoenyzme der Fructose-Phosphat-Aldolase im Serum von Ratten mit durch Diäthylnitrosamin induzierten Lebertumoren
}

\author{
Von A. L. Dikow und D. Hadjrolov
}

Aus der Biochemischen Abteilung und der Abteilung fïr Experimentalkanqerogenese des Onkologischen Forschungsinstituts (Direktor: Prof. N. Antchew), SofialBulgarien

(Eingegangen am 12. August 1969)

Die Gesamtaktivität und das Isoenzymmuster der Serumaldolase wurden an Ratten mit durch Diäthylnitrosamin induzierten primären Lebertumoren untersucht. Es werden eine wesentliche Erhöhung der Aldolaseaktivität des Serums sowie Veränderungen in ihrem Isoenzymmuster nachgewiesen. Im kathodischen Teil des Zymogramms der Serumaldolase treten vier Isoenzymfraktionen vom Typ Leber Aldolase B auf, die nach ihrer elektrophoretischen Beweglichkeit mit den vier kathodischen Fraktionen der. Tumoraldolase übereinstimmen. Solche Veränderungen im Isoenzymmuster der Serumaldolase konnten von den Verfassern bei infektiösen oder toxischen Leberschädigungen niemals nachgewiesen werden; sie haben wegen ihres spezifischen Charakters eine wichtige Bedeutung für die Tumordiagnostik.

\section{Isoenzymes of fructose phosphate aldolase in the serum of rats with liver tumours induced by diethylnitrosamine}

The total activity and isoenzyme pattern of serum aldolase were studied in rats with primary liver tumours induced by diethylnitrosamine. In comparison with normal serum, the aldolase activity was inr" tied and the isoenzyme pattern was changed. The cathodic part of the serum aldolase zymogram showed four isoenzyme fractions attri ...ble to type B liver aldolase, and these were electrophoretically identical with the four cathodic fractions of the tumour aldolase. Similar cnanges in the isoenzyme pattern of serum aldolase could not be found in any cases of infectious or toxic liver damage; in view of the specific nature of these changes, they are important in tumour diagnosis.

Warburg und Christian stellten 1943 einen „wesentlichen Anstieg der Serumaldolase bei Ratten mit transplantiertem Jensen-Sarkom" fest (1). Später beobachteten eine Reihe von Verfassern eine Erhöhung der Serumaldolaseaktivität bei Kranken mit verschiedenen Tumoren (Prostatakarzinom, Karzinom der Harnblase) und insbesondere mit Lebertumoren $(2,3)$. Da die Aldolaseaktivität im Serum auch bei anderen Leberktankheiten steigt (infektiöse und toxische Hepatitis, Metastasen von anderen Tumoren) $(4,5,6,7)$, ist die Untersuchung des Isoenzymmusters der Aldolase für die Diagnostik von großem Interesse. In vorhergehenden Mitteilungen studierten wir das Isoenzymmuster der Serumaldolase bei Menschen mit infektiöser Hepatitis (8), sowie an Ratten, deren Leber mit verschiedenen Agentien beschädigt war $(9,10,11)$. In vorliegender Arbeit untersuchten wir das Isoenzymmuster der Serumaldolase an Ratten mit Diäthylnitrosamin induzierten primären Lebertumoren.

\section{Material und Methoden}

Die Versuche wurden an männlichen Albinoratten von etwa $140 \mathrm{~g}$ Gewicht durchgeführt. Die Tiere bekamen im Trinkwasser täglich $1 \mathrm{mg}$ Diäthylnitrosamin $6 \mathrm{mal}$ wöchentlich während 165 Tagen. Am 210. Tage nach Versuchsbeginn waren die Tumoren leicht durch die Bauchwand zu palpieren. Danach wurden die Tiere durch die A. femoralis entblutet. Im Blutserum bestimmten wir die Gesamtaldolaseaktivität durch den Aldolase UV-Test, Fa. Boehringer, Mannheim. Die Isoenzyme der Aldolase trennten wir elektrophoretisch auf 0,6proz. Agarosegel mit Tris/EDTA/Borsäure-Puffer $\mathrm{pH} 8,9$ nach früher beschriebenem Verfahren (12). Die Detektion der Isoenzymfraktionen der Aldolase erfolgte nach der von Drkow vorgeschlagenen Methode (13). Zur Untersuchung wurde Material von Tumoren und vom tumorfreien Teil der Leber der Versuchstiere entnommen sowie von der Leber der Kontrolltiere. Wir homogenisierten das Material bei $0^{\circ}$, zentrifugierten $30 \mathrm{Min}$. bei $160000 \mathrm{~g}$ und $0^{\circ}$. Den klaren Uberstand benutzten wir zur Trennung der Isoenzyme nach oben genanntem Verfahren.

\section{Ergebnisse}

Im 7. Monat nach Versuchsbeginn bildeten sich in der Leber einzelne große und viele kleinere Tumoren (Abb. 1). Diese stellten histologisch hepatozelluläre Karzinome dar, deren Zellen in bezug auf Größe, Intensität der cytoplasmatischen Basophylie und ihrer mitotischen Aktivität variierten. Im Tumorparenchym konnten öfters einzelne oder gruppierte Zellnekrosen beobachtet werden, wobei in einzelnen Fällen auch makroskopisch nektrotische Bereiche an den Tumoren festgestellt werden konnten. Die Gesamtaldolaseaktivität im Serum der sechs untersuchten Ratten mit primären Lebertumoren betrug durchschnittlich $115,5 \mathrm{mU} / \mathrm{ml}$, während wir bei den Kontrolltieren $54,6 \mathrm{mU} / \mathrm{m} l$ fanden.

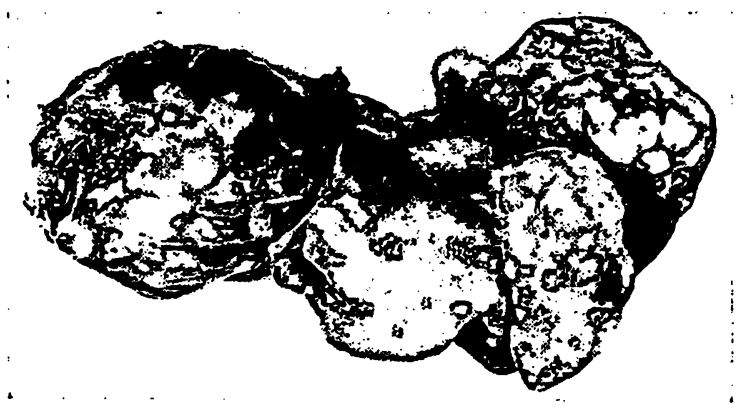

Abb. 1

Rattenleber mit Tumoren von verschiedener Größe, die Diäthylnitrosamin-induzierte primäre Leberkarzinome darstellen 


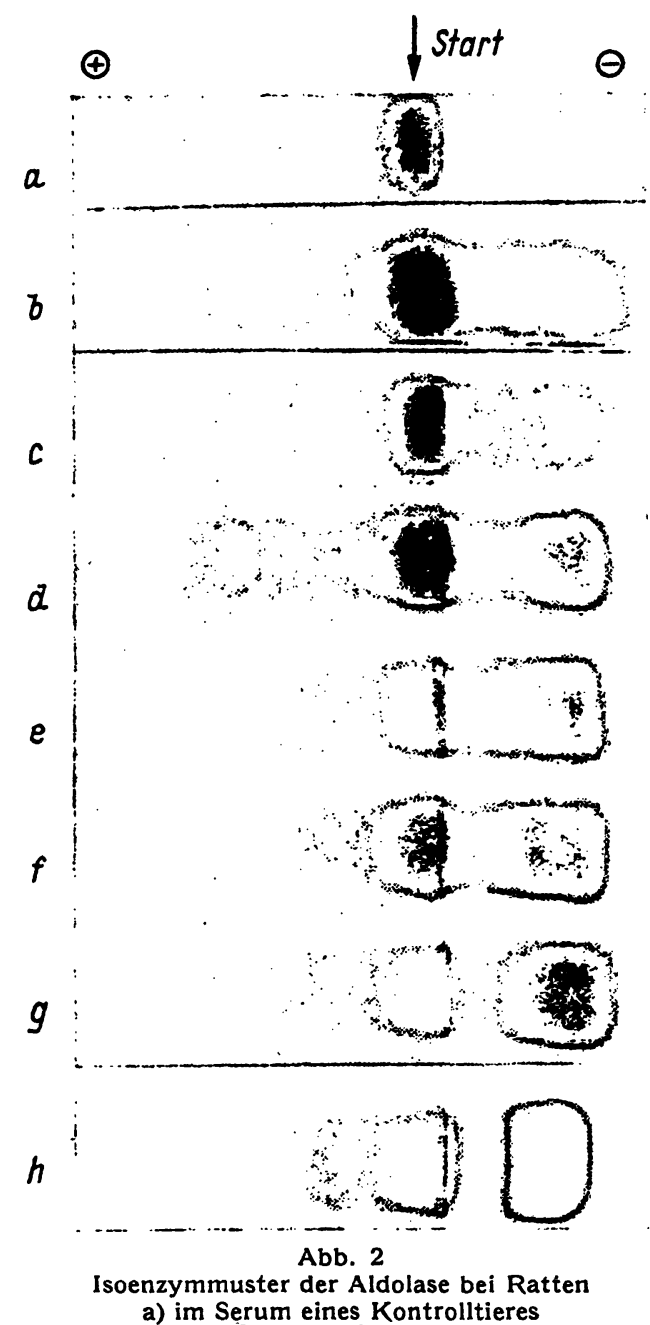

a) im Serum eines Kontrolltieres
alde

b, c, d) im Serum von Ratten mit Diäthylnitrosamin induzierten Lebertumoren

e, f) im Parenchym der primären Lebertumoren

g) im nicht vom Tumorprozeß einbezogenen Bereich der Leber eines

. h) in der Leber eines Kontrolltieres

Das Isoenzymmuster der Aldolase im Serum, in den Tumoren und der Leber ist auf Abbildung 2 dargestellt.

Bei den Kontrolltieren besteht das Isoenzymmuster der Serumaldolase aus einer intensiven Fraktion, die sich anodisch bei der Startlinie befindet und aus einer schwächeren Fraktion am anodischen Ende des Zymogramms. Außerdem befinden sich kathodisch bei der Startlinie zwei kaum bemerkbare Isoenzymfraktionen, die nach ihrer elektrophoretischen Beweglichkeit mit den entsprechenden Fraktionen der Leberaldolase übereinstimmen und vom Typ Aldolase B sind (Abb. 2 a). Das Isoenzymmuster der Serumaldolase bei Tieren mit Lebertumoren ist auf Abbildung $2 \mathrm{~b}, \mathrm{c}, \mathrm{d}$ dargestellt. Hieraus ist zu ersehen, daß die anodisch bei der Startlinie liegende Fraktion, sowie diejenige am anodischen Ende des Zymogramms eine mehrfach erhöhte Intensität besitzen. Zwischen ihnen treten außerdem zwei neue Fraktionen hervor, die in manchen Fällen ziemlich intensiv sind (Abb. 2 d). Auf der kathodischen Seite des Zymogramms erscheinen vier deutlich dargestellte und intensive Isoenzymfraktionen vom Typ Aldolase B. Im Isoenzymmuster der Aldolase in den Lebertumoren (Abb. 2 e, $f$ ) werden eine anodisch bei der Startlinie liegende Fraktion und zwei schwächere am anodischen Ende des Zymogramms nachgewiesen. Kathodisch von der Startlinie befinden sich vier Fraktionen, die ziemlich intensiv, deutlich voneinander abgesondert und vom Typ Aldolase B sind. In den Bereichen der nicht in den Tumorprozeß einbezogenen Leber der Versuchstiere sind diese vier kathodischen Isoenzymfraktionen nicht nachweisbar. Es werden nur zwei intensive kathodische Fraktionen beobachtet, die wegen ihrer nahen Lage, ähnlich wie bei der normalen Leber (Abb. 2 h), zusammenfließen (Abb. $2 \mathrm{~g}$ ).

\section{Diskussion}

An Patienten mit primären Lebertumoren wurden sehr hohe Werte der Serumaldolaseaktivität festgestellt (2). Diese Angaben stimmen mit den Ergebnissen vorliegender Arbeit bei Diäthylnitrosamin-induzierten Lebertumoren an Ratten überein. Bei Untersuchungen des Isoenzymmusters der Serum-Lactatdehydrogenase an Kranken mit verschiedenen Tumoren, erhielten $\mathrm{RICH}-$ TERICH und Mitarbeiter sehr verschiedene Ergebnisse. Daher resultiert ihre Auffassung, daß dieser Untersuchung keine diagnostische Bedeutung zugesprochen werden kann (14). Später jedoch stellten RUBERTI und Mitarbeiter (15) bei ähnlichen Untersuchungen fest, daß die Bestimmung des Isoenzymmusters der SerumLactatdehydrogenase bei Kranken mit verschiedenen Tumoren von Bedeutung für die Differentialdiagnose des Krebses ist. Dies wird von ZIEGENBEIN (16) unterstützt, nach dem vor allem das Isoenzymmuster der verschiedenen Enzyme in den Tumoren und in den entsprechenden normalen Geweben untersucht werden muß. Erst darauf können die Veränderungen im Serum gesucht werden.

Bei unseren vorhergehenden Untersuchungen über das Isoenzymmuster der Serumaldolase sowohl bei Kranken an infektiöser Hepatitis (8) als auch bei toxischer Schädigung mit verschiedenen Agentien der Leber an Ratten $(9,10,11)$ stellten wir gleichzeitig mit der beträchtlich erhöhten Serumaldolaseaktivität auch wesentliche Veränderungen in ihrem Isoenzymmuster fest. Im kathodischen Teil des Zymogramms beobachteten wir (im Vergleich zu Normalfällen) zwei kathodische Isoenzymfraktionen mit mehrfach erhöhter Intensität, die nach ihrer elektrophoretischen Beweglichkeit vollkommen mit den zwei kathodischen Fraktionen der Leberaldolase übereinstimmen. Im kathodischen Teil des Isoenzymmusters der Aldolase der untersuchten primären Lebertumoren an Ratten konnten vier Isoenzymfraktionen nachgewiesen werden, die nach ihrer elektrophoretischen Beweglichkeit und Substratspezifität gegenüber Fructose-1,6-diphosphat und Fructose-1-phosphat vom Typ Leber Aldolase B sind (17). Im Isoenzymmuster der Serumaldolase bei diesen Ratten waren vier kathodische Fraktionen vom Typ Aldolase B nachweisbar, die nach ihrer elektrophoretischen Beweglichkeit vollkommen mit den kathodischen Fraktionen der Aldolase von den Tumoren übereinstimmen, aus der sie auch offensichtlich hervorkommen. Dadurch wird bewiesen, $\mathrm{da}$ ein wesentlicher Teil der Serumaldolase bei Ratten mit primären Lebertumoren aus jenen Tumoren 
stammt, deren Zellen infolge schnellen Wachstums nud schwacher Blutzufuhr leicht geschädigt werden und nekrotisieren. Die Veränderungen im anodischen Teil des Zymogramms der Serumaldolase an Ratten mit Lebertumoren beobachtet man auch bei infektiösen und toxischen Leberschädigungen. Sie sind wahrscheinlich auf die Einbeziehung auch von anderen Organen in den pathologischen Vorgang zurückzuführen.
Die unsererseits nachgewiesenen Veränderungen im Isoenzymmuster der Serumaldolase an Ratten mit primären Lebertumoren sind bei anderen Leberschädigungen nicht festzustellen. Ihres spezifischen Charakters wegen haben sie eine wichtige Bedeutung für die Tumordiagnostik.

\section{Literatur}

1. Warburg, O. und W. Christian, Biochem. Z. 314, 399 (1943). 2. Krussanowa, N. I. und A. I. KRassowskaja, Vopt. onkol., UdSSR, 9, 9 (1963). - 3. SaragoçA, A., Amer. J. Digest. Dis. 9, 337 (1964). - 4. BRUNs, F. und W. Puls, Klin. Wschr. 32, 656 (1954). - 5. Bruns, F. und W. JACOB, Klin. Wschr. 32, 1041 (1954). - 6. TAN, C., Cancer 16, 1373 (1963). - 7. Siblex, J., G. Higgins und G. Fleischer, Amer. Med. Ass. Arch. Pathol. 59, 715 (1955). - 8. Dikow, A. L. und M. Romanow, Zschr. ges. Inn. Med. 23, 471 (1968). - 9. Drkow, A. L. und D. HaDjrorov, diesé
Z. 7, 160 (1969), - 10. Dikow, A. L. und D. HadjroLov, diese Z. 7,556 (1969). - 11. Dikow, A. L. und D. HaDjrolov, diese Z. 8,35 (1970). - 12. Dikow, A. L. und V. Genowa, diese Z. 7, 155 (1969). - 13. Drkow, A. L., diese Z. 6, 386 (1968). 14. RuPpINGER, K., R. Richterich und E. Rossi, Schweiz med.. Wschr. 92, 198 (1962). - 15. Rựertr, A., E. Castellani und G. Tobalpini, Arcispedale S. Anna Ferrara, 19, 1079 (1966). - 16. ZiegendeIn, R., Klin. Wschr. 43, 1337 (1965). - 17. Hadjrolov, D. und A. L. Drkow, Zschr. Krebsforsch. (1970) in Druck.
Dr. med. Angel L. Dikow Ruhr-Universität Lehrstuhl Biochemie 4630 Bochum-Querenburg Postfach 2148 


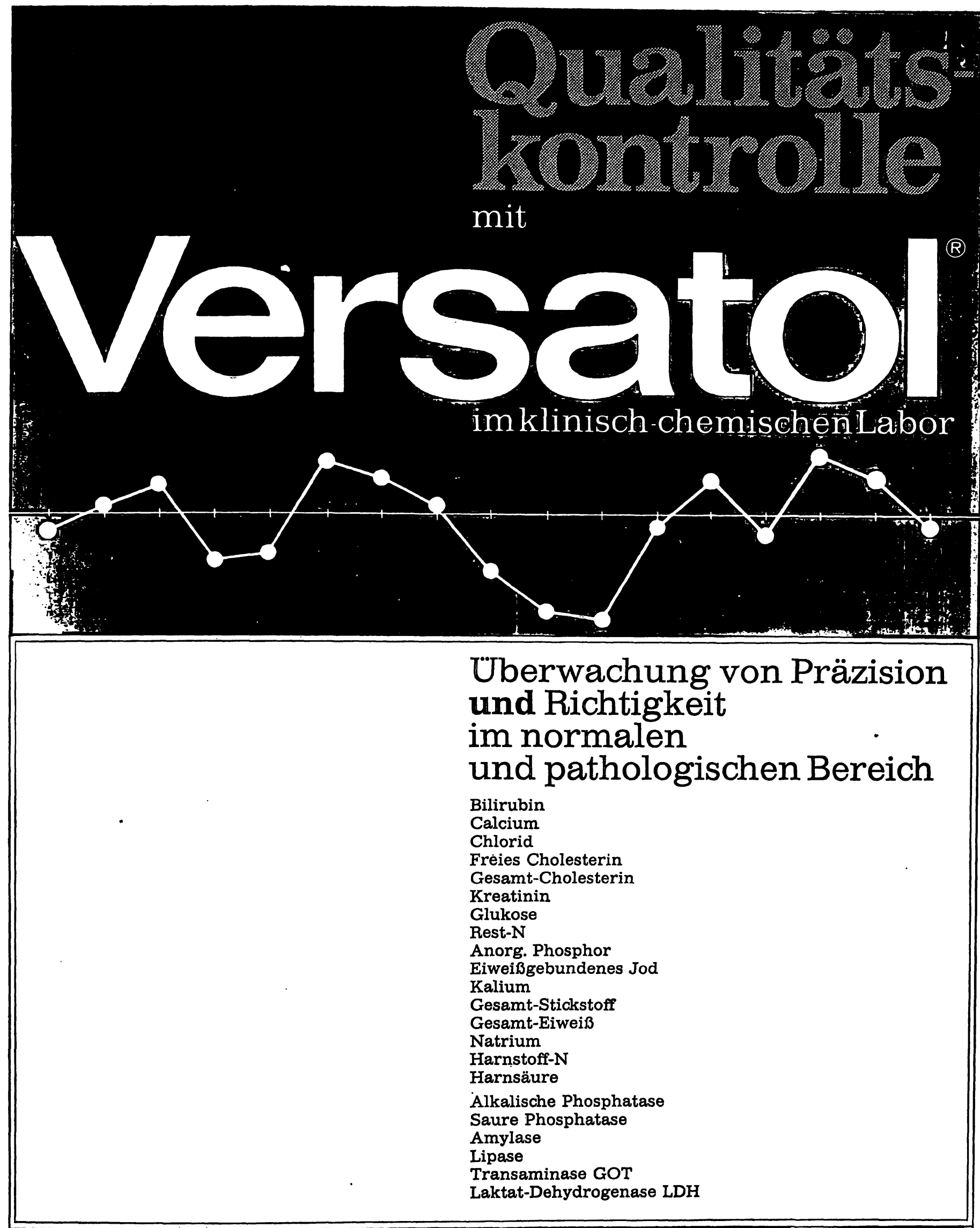

\section{GODECKE}




\section{Technicon \\ Symposium 70}

\section{Kurhaus}

Bad Homburg v.d. Höhe 29./30. Januar 1970

\author{
* Biochemische Vielfachanalyse \\ * Klinische Chemie \\ * Hämatologie, Immunologie, Serologie \\ * Chromatographie
}

Deutsche und ausländische Wissenschaftler werden am 29. und 30. Januar 1970 in Bad Homburg v.d.H. über die Errgebnisse inrer Arbeit in der Automation klinisch-chemischer, hämatologischer, immunologischer, serologischer und chromatographischer Analysenverfahren berichten.

Dabei werden die Vorträge und Diskussionen um die Erstellung biochemischer Profile einen wichtigen Platz einnehmen.

Das Kernstück eines modernen Laboratoriums wird im Routinebetrieb demonstriert.

Tagungsunterlagen senden wir auf Anforderung gerne zu.

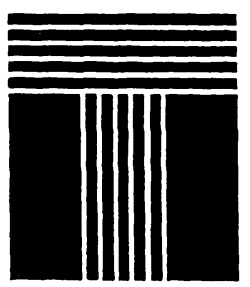

Technicon GmbH 6 Frankfurt (Main) Sternstraße 8

Tel. 0611/590221-3

\section{Zum fehlerfreien} Photographieren von DünnschichtChromatogrammen CAMAG REPRO-STAR

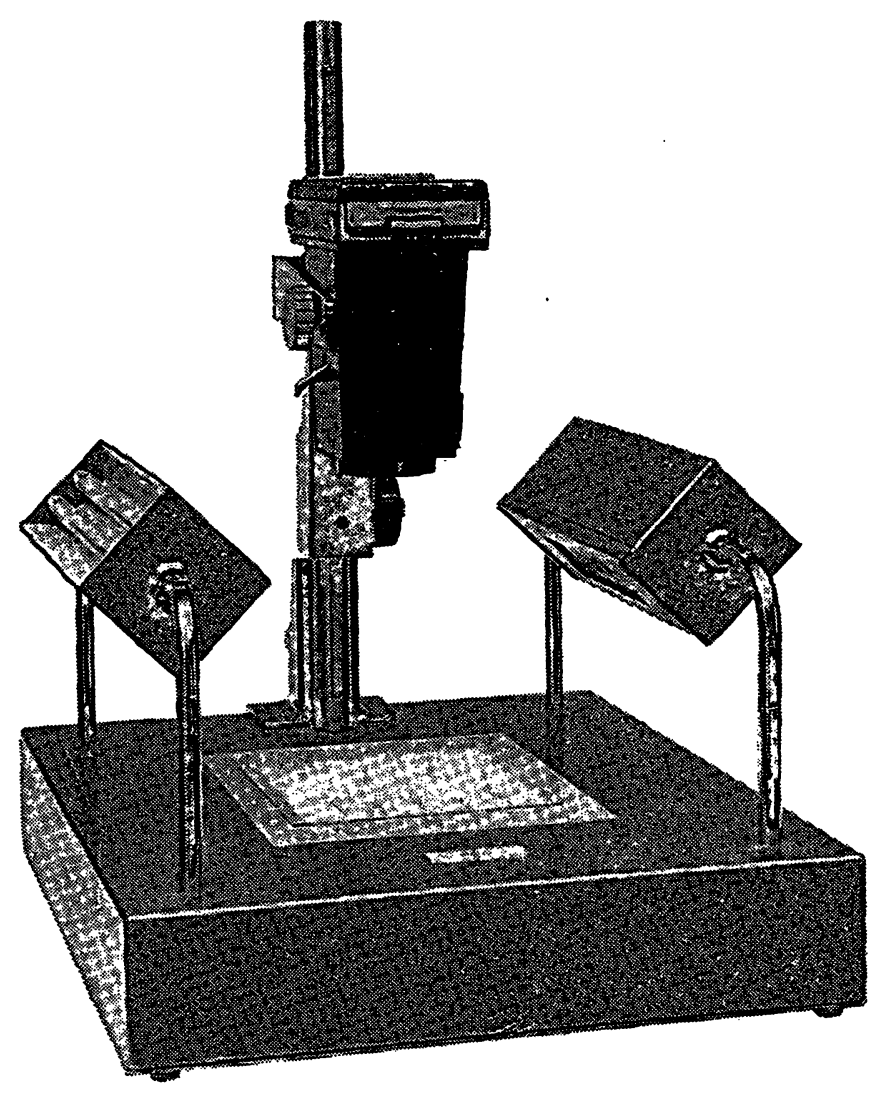

Mit dem CAMAG REPRO-STAR

photographieren Sie unter langwelligem UV, kurzwelligem UV und im sichtbaren Licht, im Auflicht- oder im Durchlichtverfahren.

Der REPRO-STAR ist so konstruiert, dass jede Aufnahme einwandfrei gelingt: Richtige Belichtungszeit, richtige Blende, richtige Scharfeinstellung und zwar mit jeder Kamera. (Ein Kamerawechsel dauert nur Sekunden.)

Verlangen Sie unser ausführliches Angebot.

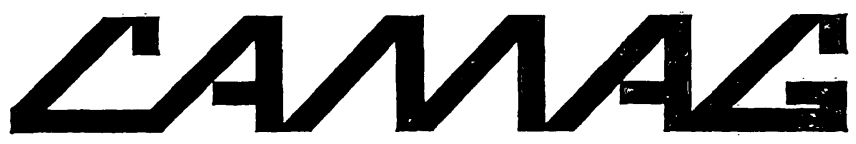

Homburgerstrasse 24 4132 Muttenz/Schweiz

Unser Zweigbetrieb in der Bundesrepublik:

1 Berlin 45, Baseler Strasse 65

Führend in Dünnschicht-Chromatographie Dünnschicht-Elektrophorese TL 39 